\title{
BMJ
}

\section{Transmission of pandemic A/H1N1 2009 influenza on passenger aircraft: retrospective cohort study}

\author{
Michael G Baker, associate professor, ${ }^{1}$ Craig N Thornley, medical officer of health, ${ }^{2}$ Clair Mills, senior lecturer, ${ }^{3}$ \\ Sally Roberts, microbiologist, ${ }^{4}$ Shanika Perera, medical officer of health, ${ }^{2}$ Julia Peters, medical officer of \\ health, ${ }^{2}$ Anne Kelso, director, ${ }^{5}$ lan Barr, deputy director, ${ }^{5}$ Nick Wilson, associate professor ${ }^{1}$
}

\begin{abstract}
'Department of Public Health, University of Otago, Box 7343 Wellington South 6242, New Zealand

${ }^{2}$ Auckland Regional Public Health Service, Auckland District Health Board, Auckland 1150, New Zealand

${ }^{3}$ Faculty of Medical and Health Sciences, University of Auckland, Auckland

${ }^{4}$ Department of Microbiology, Auckland District Health Board PO Box 110031, Auckland, New Zealand
\end{abstract}

${ }^{5}$ WHO Collaborating Centre for Reference and Research on Influenza, North Melbourne, Victoria 3051, Australia

Correspondence to: M Bake michael.baker@otago.ac.nz

Cite this as: BMJ 2010;340:c2424 doi:10.1136/bmi.c2424

\section{ABSTRACT}

Objectives To assess the risk of transmission of pandemic A/H1N1 2009 influenza (pandemic A/H1N1) from an infected high school group to other passengers on an airline flight and the effectiveness of screening and follow-up of exposed passengers.

Design Retrospective cohort investigation using a questionnaire administered to passengers and laboratory investigation of those with symptoms.

Setting Auckland, New Zealand, with national and international follow-up of passengers.

Participants Passengers seated in the rear section of a Boeing 747-400 long haul flight that arrived on 25 April 2009 , including a group of 24 students and teachers and 97 (out of 102) other passengers in the same section of the plane who agreed to be interviewed.

Main outcome measures Incidence of in-flight infection with pandemic $\mathrm{A} / \mathrm{H} 1 \mathrm{~N} 1$; sensitivity and specificity of influenza symptoms for confirmed infection; and completeness and timeliness of contact tracing. Results Nine members of the school group were laboratory confirmed cases of pandemic A/H1N1 infection and had symptoms during the flight. Two other passengers developed confirmed pandemic $\mathrm{A} / \mathrm{H} 1 \mathrm{~N} 1$ infection, 12 and 48 hours after the flight. They reported no other potential sources of infection. Their seating was within two rows of infected passengers, implying a risk of infection of about $3.5 \%$ for the 57 passengers in those rows. All but one of the confirmed pandemic $\mathrm{A} / \mathrm{H} 1 \mathrm{~N} 1$ infected travellers reported cough, but more complex definitions of influenza cases had relatively low sensitivity. Rigorous follow-up by public health workers located $93 \%$ of passengers, but only $52 \%$ were contacted within 72 hours of arrival.

Conclusions A low but measurable risk of transmission of pandemic $\mathrm{A} / \mathrm{H} 1 \mathrm{~N} 1$ exists during modern commercial air travel. This risk is concentrated close to infected passengers with symptoms. Follow-up and screening of exposed passengers is slow and difficult once they have left the airport.

\section{INTRODUCTION}

The containment phase of the New Zealand (NZ) pandemic influenza plan proposes that arriving airline passengers with suspected influenza and their in-flight contacts should be identified and managed to reduce the risk of importation of disease. ${ }^{1}$ The pandemic plans of some other countries include similar measures. ${ }^{2}$ However, little documented evidence exists of influenza transmission during flights. A recent review identified only three "influenza" outbreaks on aircraft. ${ }^{3}$ One was not laboratory confirmed, ${ }^{4}$ and the other two occurred before regulations on ventilation for commercial aircraft were published. ${ }^{56}$

On 25 April 2009 the World Health Organization declared the outbreak of novel pandemic A/H1N1 2009 influenza (pandemic A/H1N1) to be a "public health emergency of international concern." "On that same day, a general practitioner in NZ identified cases of influenza-like illness in a group of high school students who, returning from a three week trip to Mexico, had disembarked off a flight from Los Angeles to Auckland six hours previously. Twelve of the group reported symptoms of influenza during the flight, and nine of these were later confirmed as NZ's first cases of pandemic $\mathrm{A} / \mathrm{H} 1 \mathrm{~N} 1$. After the termination of the flight, two further members of the group and three passengers outside the group developed symptoms of influenza. This timing raised the possibility that these were cases of in-flight infection with influenza.

This investigation therefore aimed to assess the risk of transmission of pandemic $\mathrm{A} / \mathrm{H} 1 \mathrm{~N} 1$ on this long haul flight to NZ. Secondary aims were to assess the effectiveness of potential questions about symptoms for identifying cases at the border and the effectiveness of contact tracing measures. The overall goal was to inform border control methods for use during the containment phase of pandemic management.

\section{METHODS}

Study design, participants, and main outcome measures This study was a retrospective cohort investigation of the risk of transmission of influenza during a commercial airline flight. The cohort consisted of all passengers seated in the rear section of the aircraft, with a further susceptible subset identified on the basis of interviewing and testing for pandemic $\mathrm{A} / \mathrm{H} 1 \mathrm{~N} 1$. The main outcome measure was the incidence of in-flight 


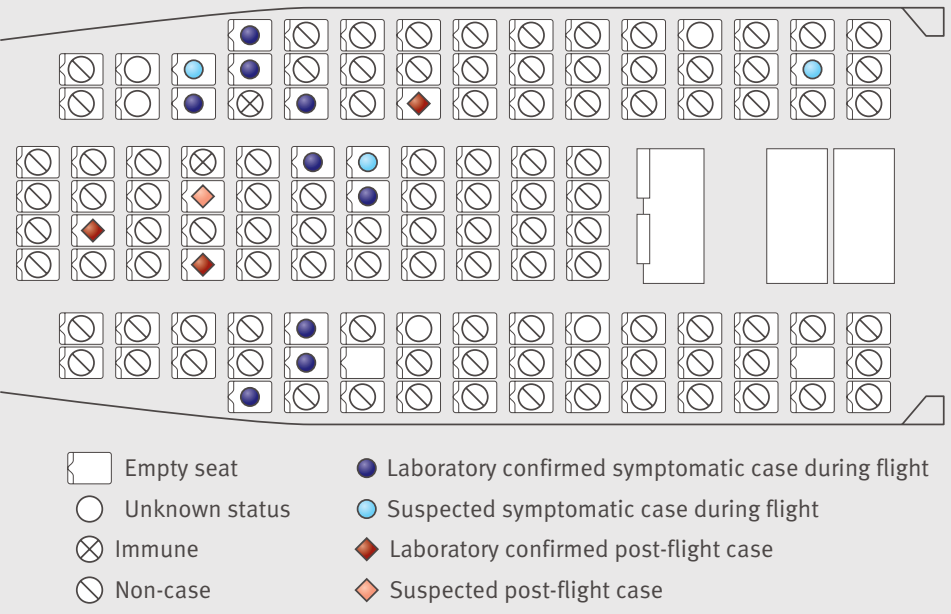

Seating plan of rear section of aircraft showing passengers according to their infection category and seating position

infection with pandemic A/H1N1. Secondary outcome measures were the sensitivity and specificity of symptoms of influenza for identifying laboratory confirmed cases and the completeness and timeliness of contact tracing.

\section{Initial public health response}

The initial public health response focused on controlling transmission from the high school group. Nasopharyngeal swabs were obtained from those with symptoms of influenza. After identification of influenza $\mathrm{A}$ in members of the group, the decision was made to trace all passengers on the flight and manage them to contain the spread of influenza (with the recommended protocol including nasopharyngeal swabs from those with symptoms, home isolation or quarantine, and antiviral post-exposure prophylaxis or treatment as appropriate). A flight manifest was obtained from the airline and arrival cards from immigration authorities in Auckland to identify passengers and their onward travel plans. Details were circulated to $\mathrm{NZ}$ public health units in the respective districts or international destinations in receiving countries.

\section{Follow-up investigation}

A follow-up investigation began during the week of 4 May 2009. All members of the high school group were interviewed with a standard questionnaire covering illness and history of symptoms before, during, and after the flight. Follow-up serological specimens were also collected from the student group 16-23 days after the flight.

The affected students were seated in the rear section of the aircraft, so this population became the focus of the retrospective cohort study. We used the seating plan and passenger lists to construct a record of all passengers in this section. We retrieved the following data on these passengers from public health units involved in the initial response: symptoms during and after the flight, results of any laboratory testing for pandemic
A/H1N1, timeliness and types of public health management. We re-interviewed passengers who reported symptoms, and those for whom no symptom history was recorded, by using a standard questionnaire. For assessment of the public health response, we defined passengers as having been "in transit" if they departed for another international destination within 24 hours of arrival or as NZ residents or visitors according to information collected during immigration processing. We used EpiInfo version 3.5.1 to analyse data.

The airline followed up cabin crew by using the same protocol as we used for passengers. We did not use results from this group in this study because we wanted to link the presence or absence of in-flight infection to seating locations, which were not applicable to mobile flight attendants. No illnesses were reported among cabin crew.

\section{Laboratory analysis}

We placed nasopharyngeal and throat swabs in viral transport media and tested them by a real time polymerase chain reaction matrix assay for influenza A using primers from the Centers for Disease Control and Prevention, Atlanta, USA. We used an ABI 7000 Sequence Detection System (Applied Biosystems) for amplification as follows: reverse transcription $50^{\circ} \mathrm{C}$ for 20 minutes, Taq inhibitor inactivation $95^{\circ} \mathrm{C}$ for two minutes followed by 45 cycles of polymerase chain reaction amplification, $95^{\circ} \mathrm{C}$ for 15 seconds, and $55^{\circ}$ $\mathrm{C}$ for 50 seconds. We sent specimens positive for influenza A RNA to a WHO collaborating centre for reference and research on influenza (Melbourne, Australia). Confirmatory testing was done by sequencing of the matrix, haemagglutinin, and neuraminidase genes for comparison with published influenza A sequences in GenBank or by real time polymerase chain reaction using primers that discriminate pandemic $\mathrm{A} / \mathrm{H} 1 \mathrm{~N} 1$ and seasonal influenza $\mathrm{A} / \mathrm{H} 1 \mathrm{~N} 1, \mathrm{~A} / \mathrm{H} 3 \mathrm{~N} 2$, and $\mathrm{B}$ sequences.

Sera were treated with enzymes that destroy receptors and assayed by haemagglutination inhibition using $1 \%$ turkey red blood cells for the presence of antibodies against reference viruses A/Auckland/1/2009 (pandemic A/H1N1) and A/Brisbane/59/2007 (seasonal A/H1N1) at the WHO collaborating centre. Seroconversion was defined as a fourfold rise in titre of haemagglutination inhibition between the acute and the convalescent serum or, when only one sample was available, the presence of a titre of at least 80 against A/Auckland/1/2009.

\section{Case definitions}

We considered passengers to have influenza-like illness if they had any two of fever or feverishness, cough, sore throat, or rhinorrhoea. We considered them laboratory confirmed if the nasopharyngeal swab was positive for pandemic $\mathrm{A} / \mathrm{H} 1 \mathrm{~N} 1$, serology detected specific antibodies to pandemic A/H1N1, or both.

We considered a passenger to be a post-flight case of influenza if they developed laboratory confirmed or 
Table 1|Status of 126 passengers in rear section of aircraft, according to pandemic A/H1N1 infection categories. Values are numbers (percentages)

\begin{tabular}{lcc} 
Status* & $\begin{array}{c}\text { School group } \\
(\mathbf{n}=24)\end{array}$ & $\begin{array}{c}\text { Other passengers } \\
(\mathrm{n}=102)\end{array}$ \\
Laboratory confirmed symptomatic case during flight & $9(38)$ & 0 \\
\hline Suspected symptomatic case during flight & $1(4)$ & $2(2)$ \\
\hline Immune case & $2(8)$ & 0 \\
\hline Laboratory confirmed post-flight case $†$ & $1(4)$ & $2(2)$ \\
\hline Suspected post-flight case† & $1(4)$ & 0 \\
\hline Non-case† & $10(42)$ & $93(91)$ \\
\hline Unknown status & 0 & $5(5)$
\end{tabular}

*See methods for definitions.

tCategories that formed cohort of pandemic A/H1N1 susceptible passengers used in subsequent analysis of influenza transmission risk $(n=107)$.

$\ddagger$ Comprised 86 passengers who reported no symptoms of influenza-like illness (fever or feverishness, cough, sore throat, or rhinorrhoea), seven passengers who reported symptoms but who did not meet case definition for influenza-like illness, nine passengers who met case definition but had negative nasopharyngeal swabs, and one student who was asymptomatic and had negative serology but was positive for pandemic A/H1N1 on nasopharyngeal swab. suspected influenza within a plausible incubation period after the flight. Assuming an incubation period of 0.6 to 3.2 days (based on values reported for influenza $\left.\mathrm{A}^{8}\right)$ and given a flight duration of 13 hours, this meant that illness from exposure on the flight could begin at any time after arrival in NZ to 3.2 days ( 77 hours) later.

We divided passengers into categories of pandemic $\mathrm{A} / \mathrm{H} 1 \mathrm{~N} 1$ infection on the basis of their combination of symptoms, timing of symptoms, and laboratory results, as follows.

Laboratory confirmed symptomatic case during flightInfluenza-like illness starting within two weeks before or during the flight with at least one infectious symptom (cough, sneeze, rhinorrhoea) persisting during the flight and nasopharyngeal swab or serology positive for pandemic A/H1N1.

Suspected symptomatic case during flight - Influenza-like illness starting within two weeks before or during the flight with at least one infectious symptom (cough, sneeze, rhinorrhoea) persisting during the flight and pandemic A/H1N1 not excluded (laboratory investigation for pandemic $\mathrm{A} / \mathrm{H} 1 \mathrm{~N} 1$ either not done or incomplete).

Immune case-Symptoms of influenza-like illness before the flight, no infectious symptoms related to this illness during the flight, and serology positive for pandemic A/H1N1.

Laboratory confirmed post-flight case - Influenza-like illness starting within 3.2 days of arrival in NZ and nasopharyngeal swab or serology positive for pandemic $\mathrm{A} / \mathrm{H} 1 \mathrm{~N} 1$.

Suspected post-flight case - Influenza-like illness starting within 3.2 days of arrival in NZ and pandemic A/H1N1 not excluded (laboratory investigation for pandemic $\mathrm{A} / \mathrm{H} 1 \mathrm{~N} 1$ either not done or incomplete).

Non-case-No symptoms of influenza during or after the flight, or symptoms of influenza during or after the flight and pandemic A/H1N1 excluded (laboratory investigations negative for pandemic $\mathrm{A} / \mathrm{H} 1 \mathrm{~N} 1$ ).
Unknown status - Could not be contacted or insufficient information to assign to another category.

We considered a laboratory confirmed post-flight case to be a case of in-flight infection if they had no other plausible sources of pandemic A/H1N1 infection (before or after the flight). In addition, we defined pandemic $\mathrm{A} / \mathrm{H} 1 \mathrm{~N} 1$ susceptible passengers as those who were seated in the rear section of the plane, excluding laboratory confirmed and suspected cases during the flight, immune cases, and those with unknown status. This susceptible group became the cohort for calculating the risk of in-flight infection with influenza.

\section{RESULTS}

Proportion of passengers successfully followed-up

The high school group had arrived on a Boeing 747400 with a total capacity of 379 passengers. Of the 128 seats in the rear section of the plane, 126 were occupied by passengers, including 24 by the high school group (22 students and two teachers). All members of the high school group were interviewed. Eight of the 24 gave nasopharyngeal swabs, and 23 gave serological specimens.

Of the remaining passengers in the rear section of the aircraft, information on influenza symptoms during and after the flight was collected from 95\% (97/102) as part of the initial response or during follow-up. Nasopharyngeal swabs were obtained from 26 of these passengers (14 of whom had symptoms). (See web table A for further details of laboratory testing.)

\section{Incidence of pandemic $\mathrm{A} / \mathrm{H} 1 \mathrm{~N} 1$ in passengers}

Table 1 shows the distribution of passengers according to categories of pandemic A/H1N1 infection. Out of a total of 121 passengers with known illness status nine were laboratory confirmed symptomatic cases during the flight. All were in the school group. Three further passengers with influenza-like illness and symptoms during the flight were incompletely investigated: one was a member of the school group (influenza A on nasopharyngeal swab, no pandemic A/H1N1 typing, serology not done); the remaining two were other passengers (nasopharyngeal swab testing not done). These three were categorised as suspected symptomatic cases during the flight.

Five passengers developed influenza-like illness after the flight. One of them developed symptoms six days after arriving and so was excluded. The remaining four developed symptoms within 3.2 days of arriving in $\mathrm{NZ}$ and so met the case definition for post-flight cases. Of these, three were laboratory confirmed and one had incomplete pandemic $\mathrm{A} / \mathrm{H} 1 \mathrm{~N} 1$ investigation (see web appendix for details of these individual cases).

\section{Characteristics of passengers with pandemic $\mathrm{A} / \mathrm{H} 1 \mathrm{~N} 1$}

The figure shows the seating position of all the passengers and distinguishes them according to the categories of pandemic $\mathrm{A} / \mathrm{H} 1 \mathrm{~N} 1$ infection used in table 1 . All four post-flight cases were seated within two rows of confirmed symptomatic $\mathrm{A} / \mathrm{H} 1 \mathrm{~N} 1$ cases. The nine 
Table 2|Symptoms reported by passengers in rear section of aircraft who were subsequently investigated for pandemic $\mathrm{A} /$ H1N1 infection by nasopharyngeal swab or serology $(n=46)^{\star}$. Values are numbers (percentages)

\begin{tabular}{|c|c|c|c|c|}
\hline & \multicolumn{2}{|c|}{ Pandemic A/H1N1 positive cases $(n=13)$} & \multicolumn{2}{|c|}{ Pandemic A/H1N1 negative $(n=33)$} \\
\hline & Symptoms present & Symptoms absent & Symptoms present & Symptoms absent \\
\hline \multicolumn{5}{|l|}{ Individual symptoms† } \\
\hline Feverishness & $5(38)$ & $8(62)$ & $4(12)$ & $29(88)$ \\
\hline Sore throat & $4(31)$ & $9(69)$ & $11(33)$ & $22(67)$ \\
\hline Runny nose/rhinorrhoea & $4(31)$ & $9(69)$ & $5(15)$ & $28(85)$ \\
\hline Cough & $12(92)$ & $1(8)$ & $7(21)$ & $26(79)$ \\
\hline Diarrhoea & $3(23)$ & $10(77)$ & 0 & $33(100)$ \\
\hline Headache & $4(31)$ & $9(69)$ & $4(12)$ & $29(88)$ \\
\hline Generally unwell & $4(31)$ & $9(69)$ & $3(9)$ & $30(91)$ \\
\hline \multicolumn{5}{|l|}{ Symptom combinations } \\
\hline ILI-US & $5(38)$ & $8(62)$ & $3(9)$ & $30(91)$ \\
\hline ILI-NZ§ & $8(62)$ & $5(38)$ & $9(27)$ & $24(73)$ \\
\hline At least one symptom & $12(92)$ & $1(8)$ & $13(39)$ & $20(61)$ \\
\hline \multicolumn{5}{|c|}{$\begin{array}{l}\text { ILI=influenza-like illness. } \\
\text { * Restricted to } 46 \text { passengers with laboratory investigation results; excluded seven passengers with one or more symptoms of ILI during or after fligh } \\
\text { who did not have adequate laboratory investigation to exclude pandemic A/H1N1 infection and two school group members considered immune } \\
\text { owing to previous pandemic A/H1N1 infection; included } 21 \text { passengers who were asymptomatic and one of who had a positive throat swab but } \\
\text { negative serology for pandemic A/H1N1. } \\
\text { †Excludes individual symptoms reported by only one pandemic A/H1N1 positive case (one each for sneezing, red eyes, nausea, vomiting, muscle } \\
\text { pain, and nose bleed) or not reported by any cases (shortness of breath, joint pain). } \\
\text { †Fever or feverishness plus cough or sore throat. } \\
\S A n y \text { two of fever or feverishness, cough, sore throat, and rhinorrhoea. }\end{array}$} \\
\hline
\end{tabular}

laboratory confirmed symptomatic cases during the flight and four post-flight cases were all in the 10-19 year age group. Web table B shows further demographic and travel history details of the passengers.

\section{Risk of in-flight infection with pandemic A/H1N1}

Two passengers ( $\mathrm{A}$ and $\mathrm{B}$ in the web appendix) with laboratory confirmed post-flight infection did not have another plausible source of infection and therefore met our criteria for in-flight infection. Passenger A developed symptoms of influenza 12 hours after the flight, and passenger B became ill 48 hours after arrival in NZ. Two others ( $\mathrm{C}$ and $\mathrm{D}$ in the web appendix) also became ill after the flight. One (passenger $\mathrm{C}$ ) could potentially have been infected before travel from other members of the student group and so is best described as possible in-flight infection. The timing of onset of symptoms of the other (passenger D) excluded infection before travel, but this case also remains inconclusive because the laboratory investigation was incomplete.

Table 1 summarises the susceptible cohort. We considered 107 passengers seated in the rear section of the plane to be susceptible to pandemic $\mathrm{A} / \mathrm{H} 1 \mathrm{~N} 1$ infection during the flight on the basis of excluding laboratory confirmed cases during the flight $(n=9)$, suspected cases during the flight $(n=3)$, immune cases $(n=2)$, and those with unknown status $(n=5)$. Of the susceptible population, 57 were seated within two rows of laboratory confirmed symptomatic cases during the flight.

We estimated the overall risk of in-flight infection in the rear section of the plane to be $1.9 \%$ (95\% confidence interval $0.3 \%$ to $6.0 \%$ ). For the 57 passengers sitting within two rows of the laboratory confirmed symptomatic cases the risk was higher at $3.5 \%(0.6 \%$ to $11.1 \%)$.

\section{Symptoms reported by pandemic A/H1N1 cases}

Table 2 shows the prevalence of symptoms reported by passengers in the rear section of the aircraft during the period from the start of the flight until four days after its arrival. One symptom (cough) was very sensitive $(92.3 \%)$ for pandemic $\mathrm{A} / \mathrm{H} 1 \mathrm{~N} 1$ infection, had relatively high specificity $(78.8 \%)$, and had moderate positive predictive value $(63.2 \%)$. The surveillance case definition for influenza-like illness used in the United States had low sensitivity (38.5\%), high specificity $(90.9 \%)$, and moderate positive predictive value $(62.5 \%)$. The screening case definition of influenzalike illness subsequently used for detecting potentially infected arriving passengers in NZ was more sensitive $(61.5 \%)$ than the US definition but had lower specificity $(72.7 \%)$ and positive predictive value $(47.1 \%)$.

\section{Effectiveness of contact tracing}

The New Zealand Ministry of Health introduced a contact tracing protocol shortly after this flight arrived in NZ. This protocol advised that contacts should be located rapidly and interviewed, have a nasopharyngeal swab if respiratory symptoms were present, be given a course of oseltamivir (this was a five day course regardless of symptoms), and be put into home isolation or quarantine for 72 hours from the start of oseltamivir treatment. This investigation assessed the extent and timeliness of this follow-up.

The results (table 3 ) show that follow-up was relatively complete but not particularly timely. Nearly all $(93 \%)$ of the passengers outside the initially identified school group received public health service follow-up, including four passengers followed up in Australia. Only $52 \%$ were followed up by public health services within 72 hours. Once identified, 81\% (77/95) received 
Table 3 Extent, timing, and completeness of public health follow-up of 102 exposed passengers in rear section of aircraft*. Values are numbers (percentages)

\begin{tabular}{|c|c|c|c|c|}
\hline Follow-up & $\begin{array}{l}\text { NZ residents } \\
\quad(n=74)\end{array}$ & $\begin{array}{l}\text { Visitors } \\
(n=19)\end{array}$ & $\begin{array}{l}\text { Transiting through } \\
\text { NZ }(n=9)\end{array}$ & $\begin{array}{c}\text { Total } \\
(n=102)\end{array}$ \\
\hline \multicolumn{5}{|l|}{ Public health follow-upt: } \\
\hline Yes & $74(100)$ & $18(95)$ & $3(33)$ & $95(93)$ \\
\hline No & 0 & $1(5)$ & $6(67)$ & $7(7)$ \\
\hline \multicolumn{5}{|l|}{ Timing of first contact + : } \\
\hline$<24$ hours & 0 & 0 & 0 & 0 \\
\hline 24-<48 hours & $2(3)$ & 0 & 0 & $2(2)$ \\
\hline $48-<72$ hours & $43(58)$ & $8(42)$ & 0 & $51(50)$ \\
\hline$\geq 72$ hours & $29(39)$ & $10(53)$ & $3(33)$ & $42(41)$ \\
\hline \multicolumn{5}{|l|}{ Completeness of public health follow-up: } \\
\hline Swab taken (if symptoms present) $\ddagger$ & $10(71)$ & $2(100)$ & $1(50)$ & $13(72)$ \\
\hline Home isolation or quarantine requested $\dagger$ & 73 (99) & $10(53)$ & $2(22)$ & $85(83)$ \\
\hline Oseltamivir offered $\dagger$ & $69(93)$ & $11(58)$ & $3(33)$ & $83(81)$ \\
\hline
\end{tabular}

$\mathrm{NZ}=$ New Zealand.

*Excluded school group of students and teachers.

†Percentages followed up calculated on basis of total 102 passengers.

$\ddagger$ Percentages calculated on basis of 18 passengers for whom collection of a swab was appropriate because influenza symptoms were present. extreme of the plausible incubation period. Infection during boarding or disembarking would also have been possible, although far less likely because of the relatively short periods of exposure involved.

Several potential modes of transmission of influenza exist (airborne aerosol, large droplet, direct contact, or indirect contact via contaminated surfaces and fomites). ${ }^{12}$ Their relative importance for transmission of influenza remains contentious. ${ }^{12-15}$ This investigation cannot establish the exact mode of transmission that occurred on this flight. The close proximity of passengers A and B to the infectious students would be compatible with all of these modes of transmission (including "short range aerosol transmission"15) rather than airborne aerosol transmission through the ventilation system. This pattern of transmission from nearby passengers is consistent with another reported case of transmission of pandemic A/H1N1 during a flight in June 2009, in which the infected person had sat within two rows of the source case,${ }^{16}$ as well as transmission of other respiratory agents in this environment. $^{3}$

the full recommended public health management. Follow-up was less complete for visitors and people transiting through NZ.

\section{DISCUSSION}

This investigation provides evidence that transmission of influenza can occur during modern commercial air travel. The risk seems to be concentrated among those people seated within two rows of infected passengers with symptoms, which is consistent with in-flight transmission of other respiratory infections. ${ }^{39}$ Our findings also show some of the difficulties and limitations of screening at entry to a country and public health follow-up of airline passengers during the containment stage of a pandemic response.

The direct detection of virus by molecular testing for pandemic $\mathrm{A} / \mathrm{H} 1 \mathrm{~N} 1$ virus or detection of specific antibodies to the virus in serum provided a high degree of certainty that laboratory confirmed symptomatic cases were true cases of pandemic $\mathrm{A} / \mathrm{H} 1 \mathrm{~N} 1$ infection. Alternatives to in-flight infection were unlikely. At the time of this episode, the global pandemic was at an early stage, so the numbers of infected people outside Mexico were relatively small. ${ }^{10}$ Infection in New Zealand was exceedingly unlikely, as no other cases had been detected in NZ at that time. ${ }^{11}$

The timing of onset of symptoms provides further evidence that infection occurred during the flight. If pandemic $\mathrm{A} / \mathrm{H} 1 \mathrm{~N} 1$ behaves like other influenza $\mathrm{A}$ viruses, we can assume an incubation period of about 1.4-1.9 days, with a range from about 0.6 days to 3.2 days. ${ }^{8}$ Both cases of in-flight infection with influenza would be consistent with this incubation period. Case A (symptom onset within 12 hours of returning to NZ) could have been infected during any part of the flight, although this range would also be consistent with infection before boarding. For case B (symptom onset 48 hours after arriving in NZ), infection before boarding would have been possible only at the upper

\section{Limitations of study}

A limitation of the investigation was that during the initial response phase passengers were interviewed by several personnel, so this process was not as complete and uniform as would be desirable. Some characteristics, notably symptoms, may therefore have been under-reported, which could have reduced case ascertainment. Time delays in interviewing would have produced further recall bias, again probably lowering reporting of symptoms. Incomplete laboratory testing of passengers in the rear section of the plane means that some infected passengers, particularly those with mild symptoms or who were asymptomatic, could have been missed. The course of oseltamivir offered to most of the passengers might also have suppressed symptoms in some (although treatment generally started several days after arrival, so this effect was probably small). Our case definition for in-flight transmission was conservative. On balance, the sources of error in this investigation would tend to underestimate the risk of in-flight transmission of pandemic A/H1N1.

\section{Implications of findings}

This investigation provides insights into the control measures that are needed during the containment phase of a pandemic response when air travel can rapidly disseminate new infections. ${ }^{17} 18$ Some equivocal evidence from the $2009 \mathrm{~A} / \mathrm{H} 1 \mathrm{~N} 1$ pandemic indicates that countries which implemented entry screening may have briefly delayed local transmission of this virus. ${ }^{19}$ Findings from this investigation support the practice of focusing attention on passengers seated near potentially infectious travellers, rather than following up all passengers on a flight. This episode also suggests that greater effort could be applied to exit screening to reduce the probability that travellers with symptoms board aircraft. In this instance, 10 members of the high school group had symptoms 


\section{WHAT IS ALREADY KNOWN ON THIS TOPIC}

Respiratory agents may be transmitted during airline travel, although the level of risk is poorly defined for most agents, including influenza

Screening for influenza is difficult because symptoms are variable and may be mild or absent

Very little evidence exists on the best way to follow up arriving travellers who might have been exposed to influenza during a flight

\section{WHAT THIS STUDY ADDS}

A low but measurable risk of contracting influenza from infected travellers with symptoms exists during a long haul flight and is concentrated within two rows of infected travellers

Screening for people infected with influenza is likely to be more sensitive if it uses the presence of single symptoms such as cough, rather than more complex case definitions

Where identification and management of passengers exposed to infections during a flight is warranted, this should be started before passengers leave the airport or board other flights

when they embarked at a US airport. Modelling of airport screening for pandemic influenza also supports the greater effectiveness of screening at the point of embarkation. ${ }^{20}$

This investigation does not suggest that the airline cabin environment is a high risk setting for transmission of influenza. The long-haul flight included nine infectious travellers, and it could be argued that the number of secondary cases was relatively small in comparison. This finding may reflect evidence that pandemic A/H1N1 virus has relatively low transmissibility. ${ }^{21}$ The number of in-flight infections was also somewhat lower than would be predicted using estimates from a recent quantitative microbial risk assessment of within flight transmission of influenza A (H1N1). ${ }^{22}$ However, as noted, our investigation methods would have tended to underestimate the risk.

Findings from this investigation also provide some information about the potential effectiveness of screening arriving passengers for symptoms of influenza. Although based on small numbers, one symptom (cough) seemed to be relatively sensitive for detecting cases subsequently found to be infected with pandemic A/H1N1. Combinations of symptoms (such as the NZ or US definitions of influenza-like illness) reduced the sensitivity of screening without greatly improving its positive predictive value. These findings are based on small numbers of passengers screened during the early phase of the A/H1N1 pandemic. They are also based on a single atypical flight that contained an unusually large number of cases of influenza. Larger studies are needed to assess the generalisability of these results to other influenza viruses, populations, seasons, and settings.

Similarly, this investigation provides some insights into the effectiveness of follow-up of passengers after arrival. As part of an extensive public health response, follow-up of these arriving passengers was relatively complete for residents of and visitors to NZ but less so for those transiting to other countries (mainly Australia). Nevertheless, this follow-up was not particularly timely; only $52 \%$ were followed up within 72 hours. Because this event came at the start of the pandemic, optimal systems were not in place to support public health follow-up. Subsequently, all passengers arriving in $\mathrm{NZ}$ airports were required to complete a detailed locator card.

\section{Further research needs}

Future investigations of airline transmission of influenza could be improved in several ways. In particular, collecting suitable laboratory specimens (such as convalescent serological samples) from all passengers in the same section of the aircraft-not just those with symptoms - would be useful to obtain a more valid estimate of the risk of transmission of influenza in these settings. Trying to put such interventions in perspective by assessing their overall effectiveness at delaying the introduction of pandemic influenza into more isolated countries, such as New Zealand, would also be useful, as would estimating the resources needed for such prevention and control measures.

\section{Conclusions}

This investigation suggests the existence of a low but measurable risk of transmission of pandemic influenza during modern commercial air travel. This risk is concentrated close to infected passengers with symptoms. Screening of arriving passengers for symptoms may need to focus on the presence of single symptoms (such as cough) to achieve a moderate degree of sensitivity. Follow-up of passengers can be difficult once they leave the airport, even when public health authorities mount a vigorous response. For island countries and those with limited entry points, such border control measures may have a role in delaying introduction and spread within the community during the early containment phase of a pandemic. However, the effort applied to such strategies needs to take into consideration the seriousness of the pandemic, the effectiveness of border control measures, and the resources required to operate them.

We thank Radhika Nagappan (LabPlus, Auckland District Health Board) and staff of the WHO Collaborating Centre for Reference and Research on Influenza (Melbourne) for laboratory work, the health workers involved in the public health response and data collection, and the passengers and the affected high school for their prompt reporting and cooperation with public health measures. The investigation of and response to this incident included staff from many agencies, notably public health units, district health boards, immigration, customs, Auckland International Airport Limited, and the Ministry of Health.

Contributors: MGB and CNT jointly initiated the investigation, developed the study design, managed the investigation, and drafted the paper. CM and SP managed data collection on airline passengers seated in the rear of the plane and interviewed these passengers. SR managed the laboratory testing of passengers. JP jointly initiated and managed the investigation. AK and IB jointly managed the laboratory typing of the influenza viruses and serological testing. NW jointly developed the study design. All authors read and approved the final version of the manuscript. MGB is the guarantor

Funding: This investigation was largely funded by the internal resources of the investigators' employing organisations as part of the public health response to the A/H1N1 pandemic. MGB was partly supported by a grant from the Centers for Disease Control and Prevention (USA) for research on pandemic influenza (1 U01 CI000445-01). The WHO Collaborating Centre for Reference and Research on Influenza is supported by the Australian Government Department of Health and Ageing. The funding sources had no involvement with the decision to write this paper and submit it for publication. 
Competing interests: All authors have completed the Unified Competing Interest form at www.icmje.org/coi_disclosure.pdf (available on request from the corresponding author) and declare: (1) MGB was partly supported by a grant from the Centers for Disease Control and Prevention (USA) for research on pandemic influenza (1 U01 CI000445-01); all othe authors declare no financial support for the submitted work from anyone other than their employer; (2) No financial relationships with commercial entities that might have an interest in the submitted work; (3) No spouses, partners, or children with relationships with commercial entities that might have an interest in the submitted work; (4) No non-financial interests that may be relevant to the submitted work.

Ethical approval: Northern Regional Ethics Committee, Auckland gave ethics approval.

Data sharing: No additional data available.

1 Ministry of Health. New Zealand influenza pandemic action plan. Ministry of Health, 2006.

2 McLeod M, Kelly H, Wilson N, Baker MG. Border control measures in the influenza pandemic plans of six South Pacific nations: a critical review. N Z Med J 2008;121:62-72.

3 Mangili A, Gendreau MA. Transmission of infectious diseases during commercial air travel. Lancet 2005;365:989-96.

4 Marsden AG. Outbreak of influenza-like illness related to air travel. Med J Aust 2003;179:172-3.

5 Moser MR, Bender TR, Margolis HS, Noble GR, Kendal AP, Ritter DG. An outbreak of influenza aboard a commercial airliner. Am J Epidemiol 1979;110:1-6.

6 Klontz KC, Hynes NA, Gunn RA, Wilder MH, Harmon MW, Kendal AP. An outbreak of influenza A/Taiwan/1/86 (H1N1) infections at a naval base and its association with airplane travel. Am J Epidemiol 1989;129:341-8.

7 Katz R. Use of revised international health regulations during influenza A (H1N1) epidemic, 2009. Emerg Infect Dis 2009; 15:1165-70.

8 Lessler J, Reich NG, Brookmeyer R, Perl TM, Nelson KE, Cummings DA, et al. Incubation periods of acute respiratory viral infections: a systematic review. Lancet Infect Dis 2009;9:291-300.

9 Silverman D, Gendreau M. Medical issues associated with commercial flights. Lancet 2009;373:2067-77.
10 Centers for Disease Control and Prevention (CDC). Update: novel influenza A (H1N1) virus infections-worldwide, May 6, 2009. MMWR Morb Mortal Wkly Rep 2009;58:453-8.

11 Baker MG, Wilson N, Huang QS, Paine S, Lopez L, Bandaranayake D, et al. Pandemic influenza A(H1N1)v in New Zealand: the experience from April to August 2009. Euro Surveill 2009;14:ii,19319.

12 Brankston G, Gitterman L, Hirji Z, Lemieux C, Gardam M. Transmission of influenza $\mathrm{A}$ in human beings. Lancet Infect Dis 2007;7:257-65.

13 Xie X, LiY, Chwang ATY, Ho PL, Seto WH. How far droplets can move in indoor environments-revisiting the Wells evaporation-falling curve. Indoor Air 2007;17:211-25.

14 Atkinson MP, Wein LM. Quantifying the routes of transmission for pandemic influenza. Bull Math Biol 2008;70:820-67.

15 Tellier R. Aerosol transmission of influenza A virus: a review of new studies. J Roy Soc Interface 2009;6(suppl 6):783-90S.

16 Han K, Zhu X, He F, Liu L, Zhang L, Ma H, et al. Lack of airborne transmission during outbreak of pandemic (H1N1) 2009 among tour group members, China, June 2009. Emerg Infect Dis 2009;15:1578-81.

17 Brownstein JS, Wolfe CJ, MandI KD. Empirical evidence for the effect of airline travel on inter-regional influenza spread in the United States. PLoS Med 2006;3:e401

18 Khan K, Arino J, Hu W, Raposo P, Sears J, Calderon F, et al. Spread of a novel influenza A (H1N1) virus via global airline transportation. $N$ Engl J Med 2009;361:212-4.

19 Cowling BJ, Lau LLH, Wu P, Wong HWC, Fang VJ, Riley S, et al. Entry screening to delay local transmission of 2009 pandemic influenza $A$ (H1N1). BMC Infect Dis 2010;10:82.

20 Malone JD, Brigantic R, Muller GA, Gadgil A, Delp W, McMahon BH, et al. US airport entry screening in response to pandemic influenza: modelling and analysis. Travel Med Infect Dis 2009;7:181-91.

21 Cauchemez S, Donnelly CA, Reed C, Ghani AC, Fraser C, Kent CK, et al. Household transmission of 2009 pandemic influenza A (H1N1) virus in the United States. N Engl J Med 2009;361:2619-27.

22 Wagner BG, Coburn BJ, Blower S. Calculating the potential for within flight transmission of influenza A (H1N1). BMC Med 2009;7:81

Accepted: 1 March 2010 